\title{
Principais instrumentos para avaliação da disfunção temporomandibular, parte II: critérios diagnósticos; uma contribuição para a prática clínica e de pesquisa

\author{
Main instruments for assessing temporomandibular disorders, part II: diagnostic \\ criteria; a contribution to clinicians and researchers
}

Thaís Cristina Chaves', Anamaria Siriani de Oliveira ${ }^{2}$, Débora Bevilaqua Grossi ${ }^{3}$

1 Fisioterapeuta; Ms.

2 Fisioterapeuta; Profa. Dra. do Depto. de Biomecânica, Medicina e Reabilitação do Aparelho Locomotor, FMRP/ USP (Faculdade de Medicina de Ribeirão Preto da Universidade de São Paulo, Ribeirão Preto, SP)

3 Fisioterapeuta; Profa. Associada do Depto. de Biomecânica, Medicina e Reabilitação do Aparelho Locomotor, FMRP/USP

ENDEREÇO PARA

CORRESPONDÊNCIA

Débora Bevilaqua Grossi

Depto. de Biomecânica - RAL

FMRP/USP Campus

Universitário

14049-900 Ribeirão Preto SP e-mail: deborabg@fmrp.usp.br

ApRESENTAÇÃO set. 2006

ACEITO PARA PUBLICAÇÃO ago. 2007
Resumo: Na literatura especializada, encontram-se variados instrumentos para avaliação da disfunção temporomandibular (DTM), sob a forma de índices, questionários, protocolos, escalas e critérios de diagnóstico. Este estudo, dividido em duas partes, visou caracterizar os principais instrumentos de avaliação da DTM disponíveis na literatura, para auxiliar o clínico e o pesquisador na correta escolha da ferramenta apropriada para seus objetivos clínicos ou científicos. Na parte I foram caracterizados dois índices clínicos e três questionários (anamnésicos e funcionais); e, nesta parte II, um questionário funcional e dois conjuntos de critérios diagnósticos. Os índices são ferramentas que organizam a avaliação de sinais e sintomas, pela obtenção de pontuações. Os questionários são melhor aplicados em estudos epidemiológicos. Para avaliação dos eventuais impactos da DTM nas atividades de vida diária, os questionários funcionais são mais adequados. Finalmente, os critérios permitem obter o diagnóstico da disfunção. O RDC/TMD (Research diagnostic criteria for temporomandibular disorders, Critérios diagnósticos para pesquisa em DTM) é um dos poucos instrumentos que define critérios operacionais para o diagnóstico clínico. O RDC/TMD, disponível em tradução oficial para o português, tem a maior parte de suas propriedades psicométricas e acurácia verificadas, caracterizando-se como uma das ferramentas mais apropriadas para avaliação da DTM.

DescritoREs: Avaliação; Diagnóstico; Índice de gravidade de doença; Questionários; Transtornos da articulação temporomandibular

ABSTRACT: Several instruments for assessing temporomandibular disorders (TMD) are available in literature, such as indices, questionnaires, protocols, scales, and diagnostic criteria. The purpose of this study, divided into two parts, was to characterise main tools available for TMD evaluation, so as to offer both researchers and clinicians guiding information on instrument selection according to their clinical or research needs. Two clinical indices and three (anamnestic and functional) questionnaires were presented in part I; and, here in part II, one functional questionnaire and two sets of diagnostic criteria. Indices consist of organized forms for assigning scores to signs and symptoms thus obtaining severity degrees. Questionnaires are better employed for epidemiological purposes. Functional questionnaires are better used to assess impact of TMD on daily life activities. There are few specific sets of systematised diagnostic criteria available for diagnosing TMD. The Research Diagnostic Criteria for Temporomandibular Disorders (RDC/TMD) has been partially translated into Portuguese and had its accuracy and most psychometric properties validated, hence seems the best choice for assessing TMD.

KEY wORDS: Diagnosis; Evaluation; Questionnaires; Severity of illness index; Temporomandibular joint disorders 


\section{INTRODUÇÃO}

Disfunção temporomandibular (DTM) é um termo que se aplica a alterações funcionais relativas à articulação temporomandibular (ATM) e estruturas mastigatórias associadas ${ }^{1}$, como ruídos articulares, limitações na amplitude de movimento ou desvios durante a função mandibular, que são considerados sinais de DTM, e dor préauricular, dor na ATM ou nos músculos mastigatórios, caracterizados como sintomas ${ }^{2}$.

Considerando-se que a etiologia da DTM é multifatorial, e que tal disfunção é caracterizada por um conjunto de sinais e sintomas, estes devem ser organizados de maneira clara, padronizada e operacional para avaliação e classificação correta da DTM, tanto funcional quanto diagnóstica. Os vários instrumentos para avaliação de DTM disponíveis na literatura apresentam vantagens, desvantagens e limitações, bem como aplicabilidades distintas.

Este estudo visou caracterizar os principais instrumentos de avaliação da DTM disponíveis na literatura, para auxiliar o clínico e o pesquisador na correta escolha da ferramenta apropriada para contemplar seus objetivos clínicos ou científicos. Na parte I (ver artigo neste mesmo número da revista) foram apresentados índices e questionários. Neste, discutem-se um questionário funcional e os critérios diagnósticos.

\section{METODOLOGIA}

Os instrumentos aqui apresentados resultam de uma pesquisa na base de dados PubMed de artigos recentes (publicados entre 2000 e 2007) mediante o descritor temporomandibular joint disorders associado a assessment, index e clinical diagnosis. Foram retidos os artigos referentes às ferramentas mais citadas e que contivessem a íntegra do instrumento proposto.

\section{QUESTIONÁRIO FUNCIONAL}

O Questionário de Sintomas Mandibulares e Hábitos Orais foi proposto por Gerstner et al. ${ }^{3}$ e é caracterizado por dois domínios: avaliação da dor mandibular e avaliação da função mandibular (Quadro 1). Para cada questão há cinco possibilidades de resposta, com pontuações variando entre 0 e 4 . A vantagem desse questionário é permitir avaliar ao mesmo tempo a severidade de sinais e sintomas clínicos e a severidade da limitação funcional relativa à DTM.

Foram verificados níveis de sensibilidade variando entre 90,3\% e 97,7\% e especificidade de $95,7 \%$ a $100 \%$ para valores de corte entre 5 e 9 da pontuação total do índice, para diferenciar pacientes com DTM em relação a um grupo controle ${ }^{3}$. Diferentemente do MFIQ (Questionário de Limitação Funcional Mandibular - ver parte I), não foi estabelecido para esse questionário-índice um sistema de graduação para quantificar a severidade da dor ou da limitação funcional. Assim, em estudos em que se objetiva fazer uma avaliação única da limitação funcional relativa à DTM, e não ao longo do tempo, ou ainda para aplicações não-relacionadas a pesquisa, o MFIQ pode ser uma alternativa mais adequada.

\section{CRITÉRIOS DIAGNÓSTICOS}

Uma das classificações diagnósticas disponíveis na literatura mais amplamente utilizada e aceita e para a qual foram relatados níveis de confiabilidade aceitáveis é o RDC/ TMD - Research Diagnostic Criteria for Temporomandibular Disorders ou Critério de Diagnóstico para Pesquisa em Disfunção Temporomandibular ${ }^{4}$. Este já foi formalmente traduzido para 18 línguas $^{5}$, incluindo o português* ${ }^{*}$ a parte denominada Eixo II do questionário já foi aplicada em uma amostra brasileira e, portanto, a etapa de validação de face já foi realizada ${ }^{6,7}$.

Um obstáculo crítico para o conhecimento da DTM é a falta de um critério diagnóstico padronizado para definir subtipos clínicos da disfunção. Corrigindo essa falha, o RDC/TMD agrupa um conjunto de critérios diagnósticos para pesquisa, visando permitir padronização e replicação da pesquisa sobre as formas etiológicas mais comuns de DTM (miogênicas e artrogênicas). O RDC/TMD tem abordagem biaxial, permitindo uma mensuração confiável de achados físicos no Eixo I e avaliação do status psicossocial, que envolve indivíduos com perfil de disfunção dolorosa crônica (depressão, ansiedade e relação desses fatores com outros sintomas físicos) no Eixo $\mathrm{II}^{4}$. Dessa forma, o RDC/TMD é uma das poucas ferramentas disponíveis na literatura que permite a avaliação diagnóstica da disfunção e das condições psicossociais a ela relacionadas, uma vez que a DTM é basicamente uma condição de dor crônica ${ }^{4}$.

O sistema de diagnóstico não é hierárquico e permite diagnósticos múltiplos para um único indivíduo. Os diagnósticos são divididos em três grupos: grupo I, Diagnósticos musculares (dor miofascial e dor miofascial com abertura limitada); grupo II, Deslocamentos de disco (deslocamento de disco com redução, deslocamento de disco sem redução com abertura limitada, e deslocamento de disco sem redução, sem abertura limitada); e grupo III, Artralgia, artrite, artrose (artralgia, osteoartrite da ATM e osteoartrose da ATM).

Como regra para o diagnóstico, a um indivíduo poderá ser atribuído no máximo um diagnóstico muscular (grupo I) (dor miofascial ou dor miofascial com abertura limitada, mas não ambos) para cada lado; e no máximo um diagnóstico do grupo II e um do grupo III. Os diagnósticos dentro de

* As diversas versões em diferentes línguas do RDC/TMD estão disponíveis no seguinte endereço eletrônico: www.rdc-tmdinternational.org/ index.htm. 
qualquer grupo são mutuamente exclusivos. Isso significa que, em princípio, um indivíduo pode receber desde um diagnóstico zero (sem condições diagnosticáveis) até cinco diagnósticos (um diagnóstico muscular mais um do grupo II mais um do grupo III, para cada lado). A ficha de avaliação clínica e o questionário de história estão disponíveis no site do RDC/TMD internacional (www.rdc-international.org). Para obtenção do diagnóstico, cada critério deve ser consultado nos itens da avaliação clínica e do questionário de história.

Algumas limitações foram apontadas nesse sistema de diagnóstico. Como se trata de um critério para pesquisa, pacientes com sinais e sintomas clinicamente relevantes e característicos de DTM podem não se enquadrar nas classificações do RDC/TMD. Assim, caso um paciente não apresente estalido articular recíproco em pelo menos duas de três repetições consecutivas do movimento de abertura/ fechamento da boca, este pode não ser incluído na categoria de deslocamento de disco com redução - o que não significa que o paciente não apresente deslocamento de disco com redução. Tal aspecto pode somente indicar que os sinais clínicos de DTM desse paciente não apresentam reprodutibilidade compatível com a exigida pelos padrões de avaliação do RDC/TMD.

Outro aspecto que deve ser considerado é a falta de evidência científica para alguns critérios operacionais como, por exemplo, para a utilização de uma libra (lb) para a palpação manual da ATM e estruturas intra-orais e 2 lbs para a palpação das estruturas extra-orais. Relatos sobre a especificidade e sensibilidade desses valores de pressão para a palpação manual são escassos na literatura ${ }^{8}$.

Quanto a suas propriedades psicométricas, não se encontraram na literatura trabalhos que tenham estabelecido os níveis de sensibilidade e especificidade do diagnóstico pelo RDC/TMD em amostras de pacientes com DTM que procuraram atendimento, ou seja, não há relatos sobre "reais pacientes com DTM" que tenham sido considerados como "não-portadores de DTM" por esse critério.

No entanto, este é o único sistema de diagnóstico que fornece a descrição detalhada de sua obtenção, em um manual de critérios operacionais; e, ainda, por se tratar de uma ferramenta biaxial, fornece um protocolo de avaliação detalhado que deve ser seguido para efetivação do diagnóstico (Eixo I) e um questionário estruturado para avaliação do status psicossocial do paciente com DTM (Eixo II). Os autores e membros do consórcio internacional em prol da iniciativa têm feito grandes esforços para que versões transculturalmente adaptadas sejam efetivadas em diferentes centros ${ }^{5,9-11}$, a fim de se obter uniformização dos termos. E estudos de confiabilidade têm sido conduzidos para seu estabelecimento por diferentes examinadores em diferentes locais do mundo ${ }^{5}$.

Vale ressaltar que, apesar de suas limitações, por ser um instrumento que contempla a maior parte dos aspectos que o tornam uma ferramenta viável do ponto de vista diagnóstico, o RDC/ TMD tem sido amplamente utilizado na literatura ${ }^{10,12-15}$. Assim, renomados pesquisadores em diferentes partes do mundo têm se voltado para a verificação de suas propriedades psicométricas e testes de acurácia de diagnóstico ${ }^{5,8,16,17}$.

\section{Critério de Diagnóstico Clínico (CDC/TMD)}

O Clinical Diagnostic Criteria for Temporomandibular Disorders (CDC/ TMD) ou Critério de diagnóstico clínico para a disfunção temporomandibular é um dos poucos sistemas de diagnóstico clínico disponíveis ${ }^{18}$. Este é o sistema utilizado pela Clínica de Dor Orofacial e Desordens Temporomandibulares da Universidade de Washington (Quadro 2). O aspecto mais importante desse critério, tal como do RDC/TMD, é seu caráter explicitamente clínico, ou seja, o clínico é capaz de obter um diagnóstico na ausência de testes laboratoriais sofisticados, com níveis satisfatórios de confiabilidade e validade ${ }^{19}$.

Dworkin e LeResche ${ }^{4}$, em uma revisão de literatura, consideraram o $\mathrm{CDC} / \mathrm{TMD}^{18}$ como o sistema de diagnóstico que contempla a maior parte dos aspectos metodológicos aplicáveis na pesquisa e na clínica de um instrumento, como método amostral utilizado no estudo de proposição da ferramenta; aplicabilidade clínica; confiabilidade intra e inter-examinadores; especificidade e sensibilidade da ferramenta; plausibilidade diagnóstica; sistema taxonômico abrangente; e a possibilidade de múltiplos diagnósticos.

Os autores desse instrumento chamam a atenção para o fato de que é um dos poucos critérios de diagnóstico clínico que possibilita a classificação de condições artrogênicas e miogênicas, além de múltiplos diagnósticos, ou seja, a classificação da disfunção de um paciente não fica restrita a um grupo apenas. Em geral, os sistemas de diagnóstico supervalorizam o diagnóstico de condições artrogênicas em detrimento do de condições miogênicas, quando o paciente pode apresentar ambas as condições. Por outro lado, porém, os autores não descrevem critérios operacionais para obtenção dos dados clínicos.

\section{CONCLUSÃO}

As várias ferramentas de avaliação de DTM disponíveis na literatura e aqui examinadas devem ter a utilização vinculada às esferas de avaliação do pesquisador ou clínico, ou seja, aspectos físicos, psicológicos, nível de interferência na realização das atividades de vida diária. Quando o objetivo é a obtenção de um diagnóstico, destacam-se o Critério de Diagnóstico Clínico para a DTM e o RDC/TMD. Este último, que resulta dos esforços dos maiores pesquisadores da área, tem a acurácia diagnóstica e a maior parte de suas propriedades psicométricas verificadas, caracterizando-se como uma das mais bem-estruturadas ferramentas disponíveis na literatura para avaliação da DTM. 
Quadro 1 Questionário de Sintomas Mandibulares e Hábitos Orais ${ }^{3}$ [Tradução não-oficial]

Questões relacionadas à dor mandibular

\begin{tabular}{|c|c|c|c|c|}
\hline \multicolumn{5}{|c|}{ Intensidade da dor } \\
\hline Nenhuma & Pouca & Muita & $\begin{array}{c}\text { Quase } \\
\text { insuportável }\end{array}$ & $\begin{array}{l}\text { Insuportável } \\
\text { sem alívio }\end{array}$ \\
\hline & & & & \\
\hline & & & & \\
\hline & & & & \\
\hline & & & & \\
\hline & & & & \\
\hline & & & & \\
\hline & & & & \\
\hline & & & & \\
\hline \multicolumn{5}{|c|}{ Intensidade do incômodo } \\
\hline Não & $\begin{array}{l}\text { Talvez um } \\
\text { pouco }\end{array}$ & Bastante & $\begin{array}{l}\text { Quase } \\
\text { sempre }\end{array}$ & $\begin{array}{l}\text { O tempo todo } \\
\text { sem parar }\end{array}$ \\
\hline
\end{tabular}

1 Sua mandíbula dói quando você abre bem a boca ou quando boceja?

2 Sua mandíbula dói quando você mastiga ou utiliza a mandíbula?

3 Sua mandíbula dói quando você não está mastigando ou utilizando a mandíbula?

4 Sua dor piora quando você acorda?

5 Você tem dor na frente das orelhas ou dores de ouvido?

6 Você tem dor nos músculos da mandíbula (bochecha)?

7 Você tem dor nas têmporas?

8 Você tem dor ou desconforto nos dentes?

Questões relacionadas à função mandibular

9 Suas articulações da mandíbula apresentam ruídos que incomodam você e os outros?

10 Você sente dificuldade em abrir a boca completamente?

11 Sua mandíbula fica travada fechada, ao ponto de você não conseguir abrir a boca?

12 Sua mandíbula fica travada aberta, ao ponto de você não conseguir fechar a boca?

13 Sua mordida é desconfortável?

Quadro 2 Critérios de diagnóstico clínico para DTM ${ }^{18}$ [Tradução não-oficial]

\begin{tabular}{|c|c|c|}
\hline Diagnóstico & Critérios operacionais & $\begin{array}{c}\text { Critérios opcionais } \\
\text { (sintomas que podem estar presentes com o diagnóstico) }\end{array}$ \\
\hline Mialgia tipo 1 & $\begin{array}{l}\text { - Relato de dor orofacial } \\
\text { - Dor à palpação muscular em dois ou mais } \\
\text { locais (músculos masseter, temporal, } \\
\text { tendão do temporal, supra-hióideo, região } \\
\text { do estilo-hióideo, pterigóideo lateral) } \\
\text { Em mais de um local, a dor deve ter intensidade de } \\
\text { pelo menos } 2 \text {, em uma escala de } 0 \text { a } 3\end{array}$ & $\begin{array}{l}\text { - Dor maçante nos músculos da face } \\
\text { - Sensaçáo de tensão ou rigidez nos músculos da face } \\
\text { - Dor ou fadiga nos músculos da face durante função } \\
\text { - Sintomas de ouvido - vertigo, tinitus }\end{array}$ \\
\hline Mialgia tipo 2 & $\begin{array}{l}\text { - Relato de dor orofacial } \\
\text { - Dor à palpação muscular em dois ou mais } \\
\text { locais (acima citados) } \\
\text { Em dois ou mais locais, a dor deve ter intensi-dade } \\
\text { de pelo menos 2, em uma escala de } 0 \text { a } 3\end{array}$ & Mesmos descritos acima \\
\hline $\begin{array}{l}\text { Disfunção dolorosa } \\
\text { miofascial }\end{array}$ & $\begin{array}{l}\text { Mialgia } 1 \text { ou 2, mais: } \\
\text { - ADM mandibular ativo <40 mm e ADM } \\
\text { mandibular passivo maior que o ativo em } \\
4 \text { ou mais mm }\end{array}$ & $\begin{array}{l}\text { Mesmos critérios para mialgia, mais: } \\
\text { - Sensação de mudança variável na mordida } \\
\text { - Desvio para o lado afetado durante abertura da boca } \\
\text { - Variação do comportamento da dor ao longo do dia }\end{array}$ \\
\hline
\end{tabular}

Abr = abertura; $\mathrm{ADM}=$ Amplitude de movimento; Fech = fechamento 
Quadro 2 Critérios de diagnóstico clínico para DTM ${ }^{18}$ [Tradução não-oficial] (cont.)

\begin{tabular}{|c|c|c|}
\hline Diagnóstico & \multicolumn{2}{|r|}{$\begin{array}{c}\text { Critérios opcionais } \\
\text { (sintomas que podem estar presentes com o diagnóstico) }\end{array}$} \\
\hline $\begin{array}{l}\text { Desarranjo interno } \\
\text { tipo } 1\end{array}$ & $\begin{array}{l}\text { - Estalido articular durante movimento } \\
\text { - Estalido articular durante protrusão e desvio } \\
\text { lateral } \\
\text { - Fech da boca normal com ou sem estalido }\end{array}$ & $\begin{array}{l}\text { - Desvio mandibular durante Abr da boca com } \\
\text { subseqüente correção após estalido } \\
\text { - Dor leve ocasional durante movimento } \\
\text { mandibular } \\
\text { - Estalido recíproco durante movimento }\end{array}$ \\
\hline $\begin{array}{l}\text { Desarranjo interno } \\
\text { tipo } 2\end{array}$ & $\begin{array}{l}\text { Mesmos critérios para desarranjo tipo 1, com } \\
\text { períodos breves de captura do disco durante } \\
\text { Abr da boca }\end{array}$ & $\begin{array}{l}\text { Mesmos critérios para desarranjo tipo 1, mais: } \\
\text { - Desvio durante Abr com recaptura; } \\
\text { - Amplitude de Abr da boca limitada a } 35 \mathrm{~mm} \text { ou } \\
\text { menos, durante captura e antes do estalido }\end{array}$ \\
\hline $\begin{array}{l}\text { Desarranjo interno } \\
\text { tipo } 3 \\
\text { (deslocamento de } \\
\text { disco sem redução) } \\
\text { (agudo ou crônico) }\end{array}$ & $\begin{array}{l}\text { - ADM mandibular ativo <35 mm } \\
\text { - Aumento da ADM mandibular passivo em } \\
\text { relação ao ativo de } 3 \text { ou mais mm } \\
\text { - História de redução súbita da amplitude de } \\
\text { Abr da boca } \\
\text { - História prévia de estalidos articulares, } \\
\text { desaparecimento do estalido e diminuição } \\
\text { repentina coincidente com Abr da boca }\end{array}$ & $\begin{array}{l}\text { - Deflexão durante movimento mandibular amplo } \\
\text { - Dor articular durante Abr ampla da boca ou na } \\
\text { Abr da boca passiva } \\
\text { - Dor à palpação articular } \\
\text { - Dor articular em repouso } \\
\text { - Aumento dos contatos oclusais no lado afetado }\end{array}$ \\
\hline Capsulite/Sinovite & $\begin{array}{l}\text { - Dor articular durante: } \\
\text { - palpação } \\
\text { - função } \\
\text { - Abr passiva da boca }\end{array}$ & $\begin{array}{l}\text { : Dor articular em repouso } \\
\text { : Inchaço articular } \\
\text { - Dor no ouvido }\end{array}$ \\
\hline $\begin{array}{l}\text { iramentos ou } \\
\text { mpressões } \\
\text { acionados a } \\
\text { umas }\end{array}$ & $\begin{array}{l}\text { Mesmos critérios para capsulite mais: } \\
\text { - História recente de trauma precedendo o } \\
\text { aparecimento de dor } \\
\text { - Dor nas excursões laterais para direita ou } \\
\text { esquerda, ou dor nos movimentos de } \\
\text { retrusão ou protrusão }\end{array}$ & $\begin{array}{l}\text { Mesmos critérios para capsulite mais: } \\
\text { - Inchaço articular } \\
\text { - Desvio da mandíbula durante Abr da boca } \\
\text { - Diminuição da ADM seguindo-se à dor }\end{array}$ \\
\hline $\begin{array}{l}\text { do disco ou } \\
\text { ligamento }\end{array}$ & Sem critérios diagnósticos para distinção & $\begin{array}{l}\text { - Estalido na Abr da boca } \\
\text { - Dor durante estalido } \\
\text { - Crepitação suave } \\
\text { - Dor articular durante função } \\
\text { - Captura do disco durante Abr ou Fech da boca }\end{array}$ \\
\hline $\begin{array}{l}\text { ença articular } \\
\text { generativa } \\
\text { rite/artrose com } \\
\text { ralgia }\end{array}$ & $\begin{array}{l}\text { Mesmos critérios para capsulite, mais: } \\
\text { - ausência de sinais positivos em testes } \\
\text { laboratoriais para doenças vasculares do } \\
\text { colágeno } \\
\text { - Crepitação ou rangido }\end{array}$ & $\begin{array}{l}\text { - Desvio durante movimento mandibular } \\
\text { - Dor articular sem função } \\
\text { - Mordida aberta anterior ou no lado não-afetado } \\
\text { - Evidências radiográficas de mudanças articulares } \\
\text { - Crepitação suave }\end{array}$ \\
\hline \multicolumn{2}{|c|}{$\begin{array}{ll}\text { Doença articular degenerativa } & \text { Mesmos critérios para a anterior (com } \\
\text { artrite/artrose sem artralgia } & \text { artralgia), exceto pela dor durante palpação, } \\
\text { (relacionada ao envelhecimento, } & \text { função ou movimentação articular } \\
\text { traumática ou idiopática) } & \end{array}$} & $\begin{array}{ll}\text { om } & \text { Mesmos critérios para a anterior (com } \\
\text { palpação, } & \text { artralgia), exceto pela ausência da dor à } \\
& \text { função, palpação e excursões } \\
\text { mandibulares }\end{array}$ \\
\hline $\begin{array}{l}\text { Doenças vasculares } \\
\text { do colágeno } \\
\text { (doença sistêmica } \\
\text { com envolvimento } \\
\text { local) }\end{array}$ & $\begin{array}{l}\text { Mesmos critérios para capsulite mais: } \\
\text { - Testes laboratoriais positivos para doenças } \\
\text { do sistema imune ou presença de critérios } \\
\text { clínicos requeridos para o diagnóstico de } \\
\text { doença colagenosa }\end{array}$ & $\begin{array}{l}\text { - Mordida aberta anterior } \\
\text { - Inchaço articular } \\
\text { - Mudanças radiográficas } \\
\text { - Redução na ADM mandibular } \\
\text { - Crepitação ou rangido articular durante } \\
\text { movimento mandibular } \\
\text { - Sinais sistêmicos ou periféricos de doença } \\
\text { vascular do colágeno }\end{array}$ \\
\hline
\end{tabular}

$\mathrm{Abr}=$ abertura; $\mathrm{ADM}=$ Amplitude de movimento; Fech = fechamento 


\section{REFERENCIAS}

1 American Society of Temporomandibular Joint Surgeons (ASTJS). Guidelines for diagnosis and management of disorders involving the temporomandibular joint and related musculoskeletal structures. Cranio. 2003;21(1):68-76.

2 Dworkin SF, Huggins KH, Leresche L, von Korff $M$, Howard J, Truelove E, et al. Epidemiology of signs and symptoms in temporomandibular disorders: clinical signs in cases and controls. J Am Dent Assoc. 1990;120(3):273-81.

3 Gerstner GE, Clark GT, Goulet JP. Validity of a brief questionnaire in screening asymptomatic subjects from subjects with tension-type headaches or temporomandibular disorders. Community Dent Oral Epidemiol. 1994;22(4):235-42.

4 Dworkin SF, LeResche L. Research diagnostic criteria for temporomandibular disorders: review, criteria, examinations and specifications, critique. J Craniomandibular Disord. 1992;6(4):301-55.

5 John MT, Dworkin SF, Mancl LA. Reliability of clinical temporomandibular disorder diagnoses. Pain. 2005;118(1-2):61-9.

6 Kosminsky M, Lucena LBS, Siqueira JTT, Pereira Jr FJ, Góes PSA. Adaptação cultural do questionário Research Diagnostic Criteria for Temporomandibular Disorders - Axis II para o português. J Bras Clin Odontol Int. 2004;8(43):51-61.

7 Lucena LBS, Kosminsky M, Costa L, Góes PSA. Validation of the Portuguese version of the RDC/ TMD Axis II questionnaire. Braz Oral Res. 2006;20(4):312-7.

8 Shaefer JR, Jackson DL, Schiffman EL, Anderson QN. Pressure-pain thresholds and MRI effusions in TMJ arthralgia. J Dent Res. 2001;80(10):1935-9.

9 Lobbezoo F, van Selms MK, John MT, Huggins K, Ohrbach R, Visscher CM, et al. Use of the Research Diagnostic Criteria for Temporomandibular Disorders for multinational research: translation efforts and reliability assessments in The Netherlands. J Orofac Pain. 2005;19(4):301-8.
10 Manfredini D, Chiappe G, Bosco M. Research diagnostic criteria for temporomandibular disorders (RDC/TMD) - Axis I diagnoses in an Italian patient population. J Oral Rehabil. 2006;33(8):551-8.

11 John MT, Hirsch C, Reiber T, Dworkin S. Translating the research diagnostic criteria for temporomandibular disorders into German: evaluation of content and process. J Orofac Pain. 2006;20(1):43-52.

12 Gavish A, Winocur E, Astandzelov-Nachmias T, Gazit E. Effect of controlled masticatory exercise on pain and muscle performance in myofascial pain patients: a pilot study. Cranio. 2006;24(3):184-90.

13 Fischer DJ. The association of temporomandibular disorder pain with history of head and neck injury in adolescents. J Orofac Pain. 2006;20(3):191-8.

14 Ohlmann B, Rammelsberg P, Henschel V, Kress B, Gabbert O, Schmitter M. Prediction of TMJ arthralgia according to clinical diagnosis and MRI findings. Int J Prosthodont. 2006;19(4):333-8.

15 Schmitter M, Gabbert O, Ohlmann B, Hassel A, Wolff D, Rammelsberg P, et al. Assessment of the reliability and validity of panoramic imaging for assessment of mandibular condyle morphology using both MRI and clinical examination as the gold standard. Oral Surg Oral Med Oral Pathol Oral Radiol Endod. 2006;102(2):220-4.

16 Schmitter M, Ohlmann B, John MT, Hirsch C, Rammelsberg P. Research diagnostic criteria for temporomandibular disorders: a calibration and reliability study. Cranio. 2005; 23(3):212-8.

17 Nilsson I, List T, Drangsholt M. The reliability and validity of self-reported temporomandibular disorder pain in adolescents. J Orofac Pain. 2006;20:138-44.

18 Truelove EL, Sommers EE, LeResche L, Dworkin SF, Von Korff M. Clinical diagnostic criteria for TMD: new classification permits multiple diagnoses. J Am Dent Assoc. 1992;123(4):47-54.

19 Schiffman EL, Anderson G, Fricton JR, Burton K, Schellhas K. Diagnostic criteria for intra-articular TM disorders. Community Dent Oral Epidemiol. $1989 ; 17 ; 252-7$. 